Journal for ImmunoTherapy of Cancer

\section{Rapid corticosteroid taper versus standard of care for immune checkpoint inhibitor induced nephritis: a single- center retrospective cohort study}

To cite: Lee MD, Seethapathy $\mathrm{H}$, Strohbehn IA, et al. Rapid corticosteroid taper versus standard of care for immune checkpoint inhibitor induced nephritis: a single-center retrospective cohort study. Journal for ImmunoTherapy of Cancer 2021;9:e002292. doi:10.1136/jitc-2020-002292

MDL and HS are joint first authors.

Accepted 16 March 2021
Check for updates

(c) Author(s) (or their employer(s)) 2021. Re-use permitted under CC BY-NC. No commercial re-use. See rights and permissions. Published by BMJ.

${ }^{1}$ Department of Medicine, Division of Nephrology, Massachusetts General Hospital, Harvard Medical School, Boston, Massachusetts, USA

${ }^{2}$ Department of Surgery,

Massachusetts General Hospital, Boston, Massachusetts, USA

${ }^{3}$ Department of Medicine,

Division of Hematology and Oncology, Massachusetts General Hospital, Boston, Massachusetts, USA

Correspondence to Dr Meghan E Sise; msise@partners.org

\section{ABSTRACT}

Background Current guidelines for treatment of immune checkpoint inhibitor (ICI)-induced nephritis are not evidence based and may lead to excess corticosteroid exposure. We aimed to compare a rapid corticosteroid taper to standard of care.

Methods Retrospective cohort study in patients with $\mathrm{ICl}$-induced nephritis comparing a rapid taper beginning with $60 \mathrm{mg} /$ day prednisone and tapered to $10 \mathrm{mg}$ within 3 weeks to a historical control group that began $60 \mathrm{mg} /$ day tapered to $10 \mathrm{mg}$ within 6 weeks (standard of care). Renal recovery was defined as creatinine returning to within 1.5fold baseline. The log-rank test compared the differences in time to renal recovery between the groups. We report rates of renal recovery at 30,60 and 90 days, and timing and outcomes of $\mathrm{ICl}$ rechallenge.

Results Thirteen patients received rapid corticosteroid taper and 14 patients received standard of care. Baseline characteristics were similar between groups. The median time to $\leq 10 \mathrm{mg} /$ day prednisone was 20 days (IQR 15-25) in the rapid-taper group compared with 38 days (IQR 30-58) in the standard-of-care group. There was no significant difference in the time to renal recovery between the groups, though numerically higher numbers of patients recovered by 30 days, 11 (85\%) in the rapid-taper arm versus $6(46 \%)$ in the standard of care arm. Exposure to other nephritis-causing medications (proton pump inhibitor or trimethoprim-sulfamethoxazole) during the corticosteroid taper was more common in the standard of care group, 9 (64\%) versus rapid-taper group, 2 (15\%), and was associated with longer time to renal recovery, 20 days (IQR 14-101) versus 13 days (IQR 7-34) in those that discontinued nephritis-causing medications. Fifteen (56\%) of patients were rechallenged with ICls, and only two (13\%) developed recurrent nephritis.

Conclusions Patients with $\mathrm{ICl}$-induced nephritis have excellent kidney outcomes when treated with corticosteroids that are tapered over 3 weeks.

\section{INTRODUCTION}

Acute interstitial nephritis (AIN) is the most common renal immune related adverse event (irAE) of immune checkpoint inhibitors (ICIs), found in $93 \%$ of patients who undergo biopsy for acute kidney injury (AKI) after ICIs. ${ }^{1}$ Like most other irAEs, current treatment recommendations for ICI-induced nephritis are based on expert consensus rather than empirical data. The current National Comprehensive Cancer Network (NCGN) guidelines suggest for persistent grade 2 AKI or higher (doubling of creatinine) that ICIs are discontinued and treatment with prednisone $1-2 \mathrm{mg} / \mathrm{kg}$ until the creatinine is $<1.5$-fold baseline, followed by a gradual taper over $4-6$ weeks. ${ }^{2-4}$ However, several studies have shown that ICI-induced nephritis is highly sensitive to corticosteroids with $>85 \%$ of patients experiencing full or partial remission of ICI-induced nephritis and very few patients requiring secondline immunosuppression. ${ }^{15-8}$ A multicenter study that included 138 cases of ICI-induced nephritis did not show an association between steroid dose or duration and the likelihood of treatment response. ${ }^{1}$ In addition, over the last several years it has become increasingly recognized that other medications associated with AIN, such as proton pump inhibitors, non-steroidal anti-inflammatory drugs, and antibiotics, potentially contribute to the pathogenesis of ICI-induced nephritis. ${ }^{6}$

Due to the known toxicities of prolonged corticosteroid treatment regimens and growing concern that high dose corticosteroids may decrease the antitumor effectiveness of ICIs, our onconephrology clinic began a clinical protocol of rapid corticosteroid taper paired with discontinuation of other offending AIN medications. ${ }^{9}$ We compared patient-important kidney and cancer outcomes among those treated with rapid taper to a retrospective cohort of patients with ICI-induced nephritis treated at our cancer center using the NCCN guidelines-based 


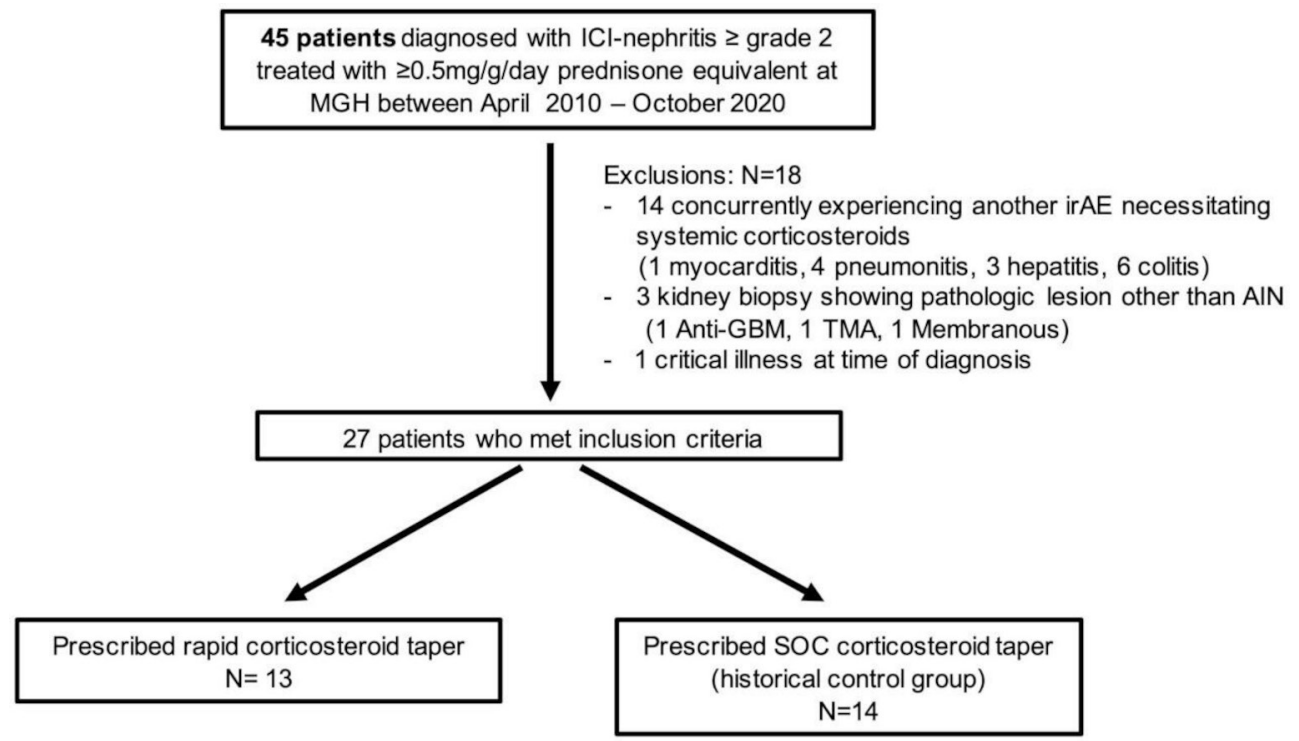

Figure 1 Patientflow. Patients who met inclusion criteria were deemed to have been eligible for "rapid- taper corticosteroids." The historical control group had been prescribed standard steroid taper by treating providers (following National Comprehensive Cancer Network guidelines). AIN, acute interstitial nephritis; GBM, glomerular basement membrane; ICI, immune checkpoint inhibitor; irAE, immune related adverse event; MGH, Massachusetts General Hospital; TMA, thrombotic microangiopathy; SOC, standard of care.

approach (standard of care) with corticosteroids tapered over 4-6 weeks.

\section{METHODS}

\section{Patients and setting}

This is a single-center retrospective cohort study conducted at Massachusetts General Hospital Cancer Center. Patients met the following criteria for ICI-induced nephritis if they had $\geq 50 \%$ increase in creatinine above their baseline creatinine at the initiation of ICI, their AKI was attributed to ICI by their treating provider, and they were treated initially with $>0.5 \mathrm{mg} / \mathrm{kg}$ prednisone equivalent per day. Because we only wanted to include control patients who could have been eligible for our rapid corticosteroid taper protocol, we excluded anyone who was concurrently experiencing another life-threatening irAE (such as myocarditis, pneumonitis, hepatitis, or colitis) as the dosage of corticosteroids needed would be driven by the other irAE. We also excluded patients who had nephrotic range proteinuria, or an alternative pathological lesion found on kidney biopsy, and those receiving care in an intensive care unit or on hospice at the time of diagnosis of ICI-induced nephritis (figure 1).

\section{Data collection}

We collected detailed clinical data on all patients using a secure, standardized, electronic case report form (REDCap). Data were manually abstracted from the medical chart for each patient, including demographics, comorbidities, use of concomitant potential AINcausing medications at baseline and time of ICI-induced nephritis, ICI doses administered, longitudinal serum creatinine and other laboratory values, kidney biopsy data, and corticosteroid treatment data. The RECIST 1.1 criteria was used to determine objective tumor response at the time of ICI-induced nephritis. ${ }^{10}$ By reviewing the electronic health record, we assessed which patients were rechallenged with an ICI after ICI-induced nephritis. We report data on rechallenge outcomes and overall survival as defined by a new complete or partial remission, or disease stabilization for $>6$ months.

\section{Determination of corticosteroid taper}

Assignment of treatment group (rapid-taper versus standard of care) was determined by retrospective chart review and was based on the initial corticosteroid taper prescribed by the treating physician. A rapid corticosteroid taper protocol was designed by two onconephrologists (HS and MES) in 2018, summarized in online supplemental table 1. All potential AIN-culprit medications were discontinued whenever possible. Patients begin therapy with $1 \mathrm{mg} / \mathrm{kg}$ /day prednisone equivalent, which was tapered to $10 \mathrm{mg}$ of prednisone equivalent within 3 weeks. Our protocol focused on quickly tapering to a dose of $10 \mathrm{mg}$ /day prednisone equivalent because this is the dosage of prednisone at which ICI rechallenge is considered per NCCN guidelines. ${ }^{2}$ The ultimate decision to rechallenge with ICIs is determined by oncologists and is based on the patient's cancer-type, level of PD1 expression by their tumor, cancer response to date, other treatment options, and patient preference. Patients in the standard of care group were prescribed a 4-6-week taper to $\leq 10 \mathrm{mg}$ of prednisone equivalent as recommended by NCCN guidelines. To mitigate selection bias, we retrospectively ascertained the treatment group based on the initially prescribed corticosteroid regimen, not the 
ultimate corticosteroid course received by the patient. Thus, patients who were prescribed a standard corticosteroid taper but were tapered rapidly due to side effects or rapid improvement remained in the standard of care group, and patients who were prescribed rapid corticosteroids taper, but ultimately required a longer corticosteroid course due to resistant or relapsing disease were still analyzed in the rapid-taper group.

\section{Primary and secondary outcomes}

Baseline creatinine was defined as the serum creatinine value just prior to initiating ICI therapy and was available for every patient. AKI severity was staged according to the Kidney Disease Improving Global Outcomes Work Group criteria. ${ }^{11}$ Our primary outcome was days to renal recovery, defined as a return of serum creatinine to $<1.5$-fold baseline creatinine. Secondary outcomes included the proportion with renal recovery by 30 and 60 days. Relapse of ICI-induced nephritis was defined as a doubling of serum creatinine that required escalation of tapered dose or re-initiation of $>0.5 \mathrm{mg} / \mathrm{kg} /$ day prednisone equivalent at any time after initially achieving recovery. Refractory nephritis was defined as a serum creatinine that did not decline to $<1.5$-fold baseline within 90 days or need for second-line immunosuppression.

We summarized outcomes in the subset of patients who were rechallenged with ICIs after recovering from ICIinduced nephritis to determine the rate of relapse of ICIinduced nephritis or new onset severe irAE that required corticosteroids. We report the median and range of days from ICI rechallenge to re-initiation of corticosteroids in patients who developed irAEs after ICI rechallenge.

\section{Statistical analysis}

We performed the statistical analyses with Stata V.16. Data are summarized by count and percentage or median and IQRs. Continuous and categorical data were compared using the Wilcoxon rank-sum and Fisher's exact tests, respectively. The probabilities of renal recovery over time, stratified by corticosteroid treatment group, were estimated using the Kaplan-Meier method. Because renal recovery is a desirable outcome, we plotted the cumulative probability of recovery rather than a survival curve. The differences between the two groups were compared using the log-rank test. All comparisons were two-tailed, with $\mathrm{p}<0.05$ considered significant.

\section{RESULTS}

Between April 2010 and October 2020, a total of 45 patients were treated with corticosteroids for ICI-induced nephritis at Massachusetts General Hospital. After applying the above exclusions, 27 patients were included in this study (figure 1). Thirteen patients were prescribed rapid corticosteroid taper and 14 historical controls received a standard of care corticosteroid taper. Table 1 shows the baseline characteristics of the two groups. The groups were overall similar at baseline in terms of
Table 1 Baseline patient characteristics

\begin{tabular}{|c|c|c|}
\hline & $\begin{array}{l}\text { Rapid taper } \\
\mathrm{N}=13\end{array}$ & $\begin{array}{l}\text { Standard of } \\
\text { care } \\
\mathrm{N}=14\end{array}$ \\
\hline \multicolumn{3}{|l|}{$\begin{array}{l}\text { Demographics median (IQR) } \\
\text { or count }(\%)\end{array}$} \\
\hline Age, years & $64(61-83)$ & $72(56-75)$ \\
\hline Male sex & $8(62)$ & $9(64)$ \\
\hline \multicolumn{3}{|l|}{ Race } \\
\hline White & $12(92)$ & $12(86)$ \\
\hline Other or unknown & $1(8)$ & $2(14)$ \\
\hline \multicolumn{3}{|l|}{ Cancer type-no. (\%) } \\
\hline Melanoma & $5(39)$ & $6(43)$ \\
\hline Lung & $3(23)$ & $1(7)$ \\
\hline Renal cell carcinoma & $1(8)$ & $3(21)$ \\
\hline Other & $4(31)$ & $4(29)$ \\
\hline \multicolumn{3}{|l|}{ ICI class - no. (\%) } \\
\hline PD-1 & $10(77)$ & $13(93)$ \\
\hline PD-L1 & $1(8)$ & $0(0)$ \\
\hline Combination CTLA4/PD-1 & $2(15)$ & $1(7)$ \\
\hline \multicolumn{3}{|l|}{ Coexisting conditions } \\
\hline Hypertension & $9(69)$ & $11(79)$ \\
\hline Diabetes & $2(15)$ & $2(14)$ \\
\hline Body mass index & $26(23-33)$ & $28(23-30)$ \\
\hline $\begin{array}{l}\text { Chronic kidney disease } \\
\left(\text { eGFR }<60 \mathrm{~mL} / \mathrm{min} / 1.73 \mathrm{~m}^{2}\right)\end{array}$ & $3(23)$ & $3(21)$ \\
\hline Baseline creatinine (mg/dL) & $0.97(0.79-1.09)$ & $0.95(0.83-1.06)$ \\
\hline Congestive heart failure & $2(15)$ & $0(0)$ \\
\hline $\begin{array}{l}\text { Chronic obstructive } \\
\text { pulmonary disease }\end{array}$ & $0(0)$ & $2(14)$ \\
\hline $\begin{array}{l}\text { History of autoimmune } \\
\text { disease }\end{array}$ & $2(15)$ & $4(29)$ \\
\hline $\begin{array}{l}\text { Prior immune related adverse } \\
\text { events events }{ }^{\star}\end{array}$ & $4(31)$ & $5(36)$ \\
\hline $\begin{array}{l}\text { Concurrent mild immune } \\
\text { related adverse events }\end{array}$ & $1(8)$ & $1(7)$ \\
\hline \multicolumn{3}{|l|}{$\begin{array}{l}\text { Findings at time of } \mathrm{ICl}- \\
\text { induced nephritis }\end{array}$} \\
\hline $\begin{array}{l}\text { Serum creatinine at } \\
\text { corticosteroid initiation }(\mathrm{mg} / \\
\mathrm{dL})\end{array}$ & $2.50(1.79-2.70)$ & $2.84(2.01-4.47)$ \\
\hline AKI stage 1 & $1(8)$ & $2(14)$ \\
\hline AKI stage 2 & $7(54)$ & $4(29)$ \\
\hline AKI stage 3 & $5(38)$ & $8(57)$ \\
\hline \multicolumn{3}{|l|}{ Leukocyte esterase } \\
\hline$\geq 1+$ leukocyte esterase & $8(62)$ & $7(50)$ \\
\hline Negative & $4(31)$ & $5(36)$ \\
\hline Not done & $1(8)$ & $2(14)$ \\
\hline $\begin{array}{l}\text { Spot urine protein to } \\
\text { creatinine ratio, median } \\
\text { (IQR)† }\end{array}$ & $0.22(0.14-0.43)$ & $0.33(0.30-0.43)$ \\
\hline Hospitalized & $5(39)$ & $4(29)$ \\
\hline Seen by a nephrologist & $13(100)$ & $13(93)$ \\
\hline
\end{tabular}


Table 1 Continued

\begin{tabular}{|c|c|c|}
\hline & $\begin{array}{l}\text { Rapid taper } \\
\mathrm{N}=13\end{array}$ & $\begin{array}{l}\text { Standard of } \\
\text { care } \\
\mathrm{N}=14\end{array}$ \\
\hline Required RRT & 0 & 0 \\
\hline $\begin{array}{l}\text { AIN diagnosed by kidney } \\
\text { biopsy }\end{array}$ & $4(31)$ & $4(29)$ \\
\hline $\begin{array}{l}\text { Concurrent use of "AIN- } \\
\text { associated" medications }\end{array}$ & $13(100)$ & $11(79)$ \\
\hline Antibiotics & $2(15)$ & 0 \\
\hline NSAIDs & $4(31)$ & $2(14)$ \\
\hline Proton pump inhibitor & $10(77)$ & $11(79)$ \\
\hline $\begin{array}{l}\text { Concurrent use of } \\
\text { nephrotoxic chemotherapy } \\
\text { cisplatin }\end{array}$ & 0 & 0 \\
\hline Tyrosine kinase inhibitor & $1(8)$ & $1(7)$ \\
\hline \multicolumn{3}{|l|}{$\begin{array}{l}\text { Number of } \mathrm{ICl} \text { doses } \\
\text { administered }\end{array}$} \\
\hline $\begin{array}{l}\text { Weeks from ICl initiation to } \\
\mathrm{ICl} \text {-induced nephritis }\end{array}$ & $8(4-10)$ & $7(3-10)$ \\
\hline $\begin{array}{l}\text { Cancer Response at time of } \\
\mathrm{ICl} \text {-induced nephritis }\end{array}$ & $27(12-42)$ & $16(11-39)$ \\
\hline Too early to determine & $2(15)$ & $4(29)$ \\
\hline Progression & $2(15)$ & $2(14)$ \\
\hline Stable disease & $1(8)$ & $3(21)$ \\
\hline Partial response & $6(46)$ & $5(36)$ \\
\hline Complete response & $2(15)$ & 0 \\
\hline
\end{tabular}

${ }^{*}$ Concurrent mild immune related adverseevent (irAE) included one case of concurrent thyroiditis and one case of concurrent adrenal insufficiency. The fraction with a concurrent irAE at the time of IClinduced nephritis is lower than prior published reports because all patients with potentially life threatening irAEs (myocarditis, hepatitis, colitis, pneumonitis, neurological irAE) were excluded from this series. †Urine protein to creatinine ratio was missing in 14 of 27 patients. AIN, acute interstitial nephritis; CTLA4, cytotoxic T-lymphocyteassociated protein 4; eGFR, estimated glomerular filtration rate; $\mathrm{ICl}$, immune checkpoint inhibitor; NSAIDs, non-steroidal anti-inflammatory drugs; PD-1, programmed death 1; PD-L1, programmed death ligand 1 ; RRT, renal replacement therapy.

gender and race, with slightly older patients in the standard of care group. Overall, medical comorbidities were common, including hypertension in $74 \%$, diabetes in $15 \%$, and chronic kidney disease (estimated glomerular filtration rate $<60 \mathrm{~mL} / \mathrm{min} / 1.73 \mathrm{~m}^{2}$ ) in $22 \%$. Baseline kidney function was similar between the groups. Use of concomitant medications associated with AIN, including proton pump inhibitors, antibiotics, and non-steroidal anti-inflammatory drugs was extremely common $(88 \%$ concurrently received at least one). Baseline use of medications associated with AIN at the time of ICI initiation was common, occurring in 18 of 27 patients $(67 \%)$. At the time of diagnosis of ICI-induced nephritis, 24 of 27 (89\%) were receiving a concurrent "AIN-associated" medication, including all patients 13 of $13(100 \%)$ in the rapid-taper group and 11 of $14(79 \%)$ in the standard of care taper group. About one-third of patients had experienced a prior irAE attributed to ICI. The majority of patients were being treated with programmed death 1 (PD-1) inhibitors at the time of ICI-induced nephritis; two patients in the rapid-taper group and one patient in the standard of care taper group were receiving combination therapy with a PD-1 inhibitor (nivolumab) and cytotoxic T-lymphocyteassociated protein 4 inhibitor (ipilimumab). The median time to ICI-induced nephritis, ICI doses received, and tumor response status at the time of ICI-induced nephritis are shown in table 1.

The findings at the time of ICI-induced nephritis were also similar between groups (table 1 ). The creatinine on the date of beginning corticosteroids was numerically higher in the group receiving standard of care steroids (2.84 mg/dL, IQR 2.01-4.47) compared with the rapidtaper group (2.50 mg/dL, IQR 1.79-2.70); it was not statistically different $(\mathrm{p}=0.65)$. The majority of patients had stage 2 or 3 AKI (24 of 27 cases, 89\%). There were more cases with stage $3 \mathrm{AKI}$ in the standard of care steroid group compared with the rapid-taper group (8 cases vs 5 cases, respectively). Median proteinuria was similar, and $\geq 1+$ leukocyte esterase was detected in the majority who had urinalysis performed. Rate of hospitalization for ICIinduced nephritis and the rate of biopsy diagnosis was also similar between the rapid taper and historical standard of care control group.

Table 2 shows the differences in corticosteroids regimens received by each group. Patients in the rapid-taper group were less likely to receive an intravenous methylprednisolone bolus at the beginning of therapy. Both groups began with $60 \mathrm{mg}$ of daily prednisone equivalent as the first oral dose, the rapid taper group was tapered to $\leq 10 \mathrm{mg}$ by a median of 20 (IQR 15-25) days. In contrast, the standard of care corticosteroid group was tapered to $\leq 10 \mathrm{mg}$ by a median of 38 days (IQR 30-58), $\mathrm{p}=0.004$. Patients were followed for a median of 461 days (IQR 300-866). Of the overall cohort, all patients but one had at least one repeat creatinine within 30 days, and all patients had at least one repeat creatinine within 60 days. There were no statistically significant differences in time to renal recovery by a log-rank test, shown in figure 2, though there were numerically higher rates of recovery by 30 and 60 days in the rapid-taper group (table 2). Overall, no patients required renal replacement therapy, and no patients required second-line immunosuppressive therapies.

In the rapid-taper group, patients were followed for a median of 356 days (83-411). Median time to renal recovery was 11 days (IQR 7-18) (table 2). Eleven of 13 $(85 \%)$ achieved renal recovery (creatinine declining to $<1.5$-fold baseline) within 30 days and one additional patient recovered after 60 days. One patient with lung cancer who had a baseline creatinine of $0.58 \mathrm{mg} / \mathrm{dL}$ had refractory ICI-induced nephritis (biopsy proven) despite being converted to a longer taper of corticosteroids; however, her creatinine remained $<1.3 \mathrm{mg} / \mathrm{dL}$ so she was not treated with second-line immunosuppression. She was not rechallenged with ICI, and fortunately had stabilization of cancer on follow-up scans nearly 11 
Table 2 Clinical outcomes

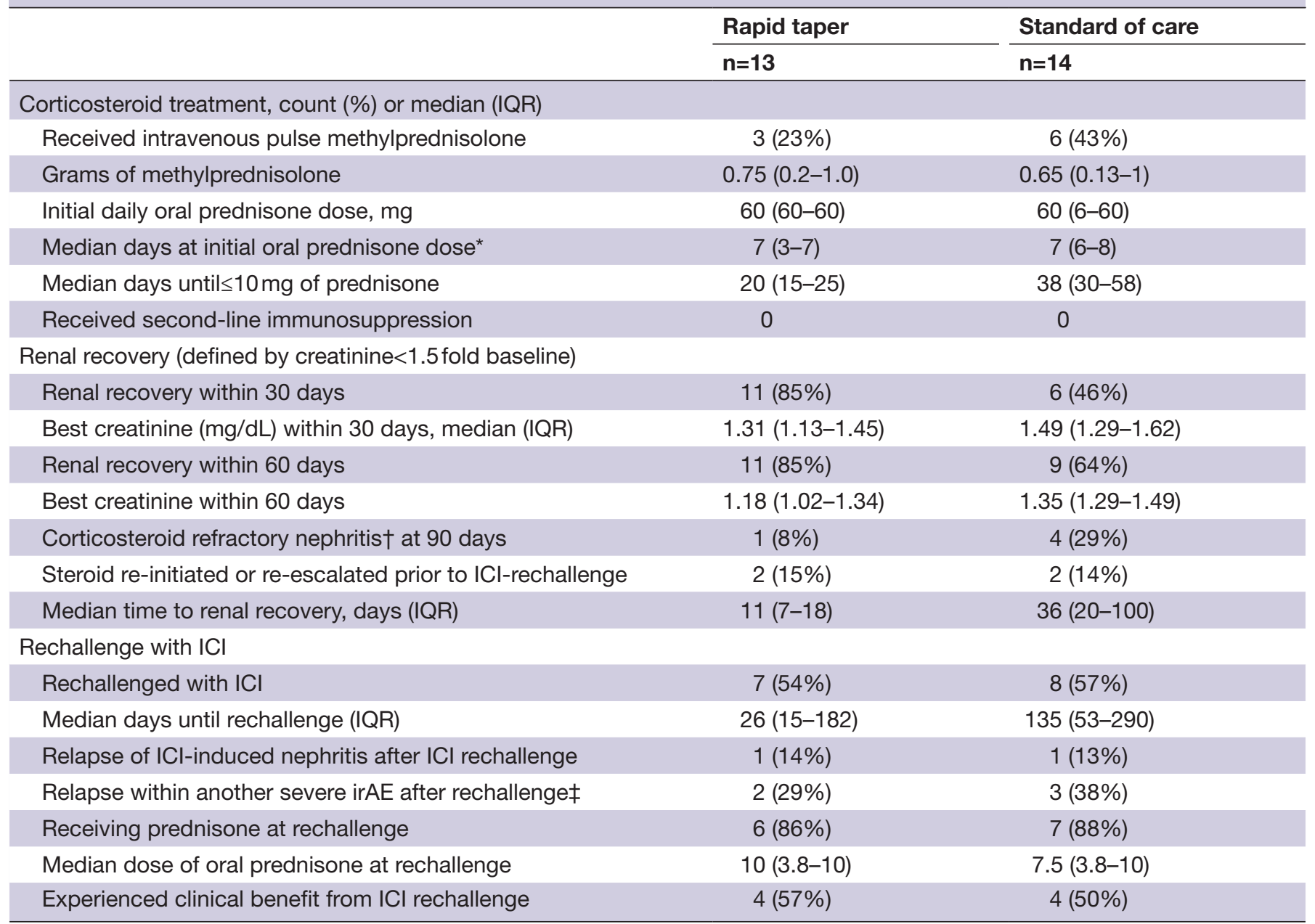

Clinical benefit from $\mathrm{ICl}$ rechallenge was defined as at least stable disease for $>6$ months.

*One patient received an oral methylprednisolone taper; the dose was converted to prednisone equivalent.

†The one patient that did not experience renal recovery at any point in the follow-up period was assigned the last follow-up date for calculating time to renal recovery. This patient had extremely low baseline creatinine $(0.58 \mathrm{mg} / \mathrm{dL})$ and her follow-up creatinine improved to 1.2 but never recovered to within 1.5 -fold baseline.

$\ddagger$ Among the patients treated with rapid taper who were rechallenged with ICls, a new severe irAE requiring corticosteroids occurred in two patients (one colitis, one pneumonitis). Among the patients treated with standard of care corticosteroid regimens who were rechallenged with $\mathrm{ICls}$, a new severe irAE requiring corticosteroids occurred in three patients (one pneumonitis, two hepatitis).

$\mathrm{ICl}$, immune checkpoint inhibitor; irAE, immune related adverse event.

months later. One patient in the rapid-taper group experienced recurrent AKI after initially recovering, and corticosteroids dose was increased; this patient died 1 month later due to perforated diverticulitis. Only two (15\%) patients in the rapid taper group received proton pump inhibitors during corticosteroid taper and none received trimethoprim-sulfamethoxazole for Pneumocystis jirovecii prophylaxis.

In the standard of care group, patients were followed for a median of 850 days (IQR 446-1277). Median time to renal recovery was 36 days (IQR 20-100). Six (46\%) of 13 patients recovered by 30 days, and 9 of 14 (64\%) achieved recovered by 60 days. Four patients had refractory nephritis at 90 days, but ultimately achieved renal recovery (range 100-227 days). In the standard of care group, $6(43 \%)$ received proton pump inhibitors during their corticosteroid taper and $5(36 \%)$ received trimethoprim-sulfamethoxazole. All four of the patients who were corticosteroid refractory at 90 days were receiving proton pump inhibitors and two of four were also receiving trimethoprim sulfamethoxazole. Across both treatment groups, there was a non-significant increase in time to recovery, 62 days (SD 21 days) in the 11 patients who had continued exposure to an AINmedications (proton pump inhibitor or trimethoprimsulfamethoxazole) compared with 24 days (SD 6 days) in the 16 patients who had all AIN medications discontinued $(\mathrm{p}=0.3)$ (online supplemental figure 1 and table 2$)$.

Over half of the patients in each group were rechallenged with ICIs after resolution of ICI-induced nephritis (table 2). Of the 15 rechallenged patients, 12 (80\%) were still receiving low dose prednisone (ranging from 2.5 to 10 


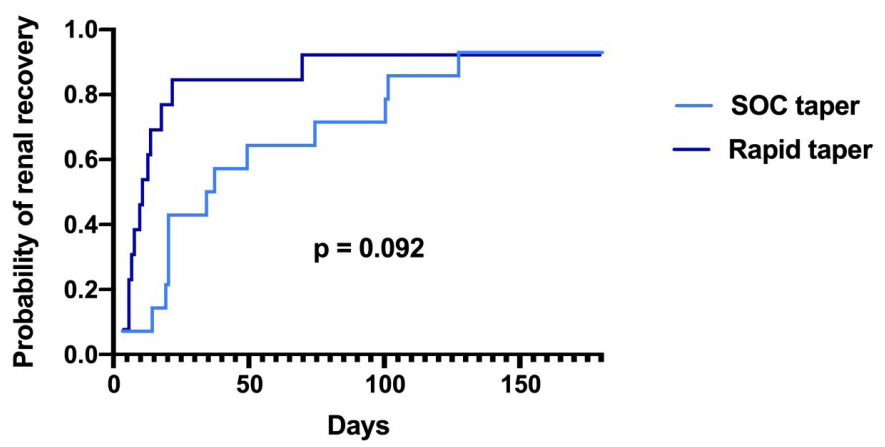

Figure 2 Time to renal recovery among patients with immune checkpoint inhibitor (ICl)-induced nephritis prescribed rapid taper. Recovery of $\mathrm{ICl}$-induced nephritis was defined as serum creatinine returning to less than 1.5 times the baseline creatinine. Curves are plotted as 1-survival probability and compared via log-rank test. Standard of care (SOC) and refers to National Comprehensive Cancer Network guidelines-based approach.

$\mathrm{mg} /$ day) at the time of ICI-rechallenge. Four of $7(57 \%)$ in the rapid-taper group and 4 of $8(50 \%)$ in the standard of care group experienced clinical benefit, defined as partial or complete remission, or disease stabilization for $>6$ months after ICI rechallenge. In each group, one patient developed recurrent ICI-induced nephritis. Two additional patients in the rapid-taper group and three patients in the standard of care group developed another severe irAE requiring corticosteroids after ICI rechallenge. Development of a new severe irAEs or relapse of ICI-induced nephritis occurred a median of 72 days after ICI rechallenge (range 53-489 days). One patient with biopsy-proven AIN, who had successfully achieved renal recovery with standard of care course of corticosteroids, was tolerating rechallenge with ICI for 5 months until he was given omeprazole for treatment of abdominal discomfort and relapsed with severe AKI 3 weeks later (figure 3).

\section{DISCUSSION}

Our single-center cohort study of patients with ICIinduced nephritis compares a rapid corticosteroid taper to historical control patients who received a corticosteroid taper per NCCN guidelines (standard of care). Our onconephrology clinic was motivated to shorten the length of corticosteroid taper given our experience and other published cohorts showing that $>85 \%$ of patients with ICI-induced nephritis respond favorably to corticosteroids and the growing concern that highdose steroids may blunt the antitumor effectiveness of ICIs. ${ }^{6-812}$ Compared with historical controls, we found

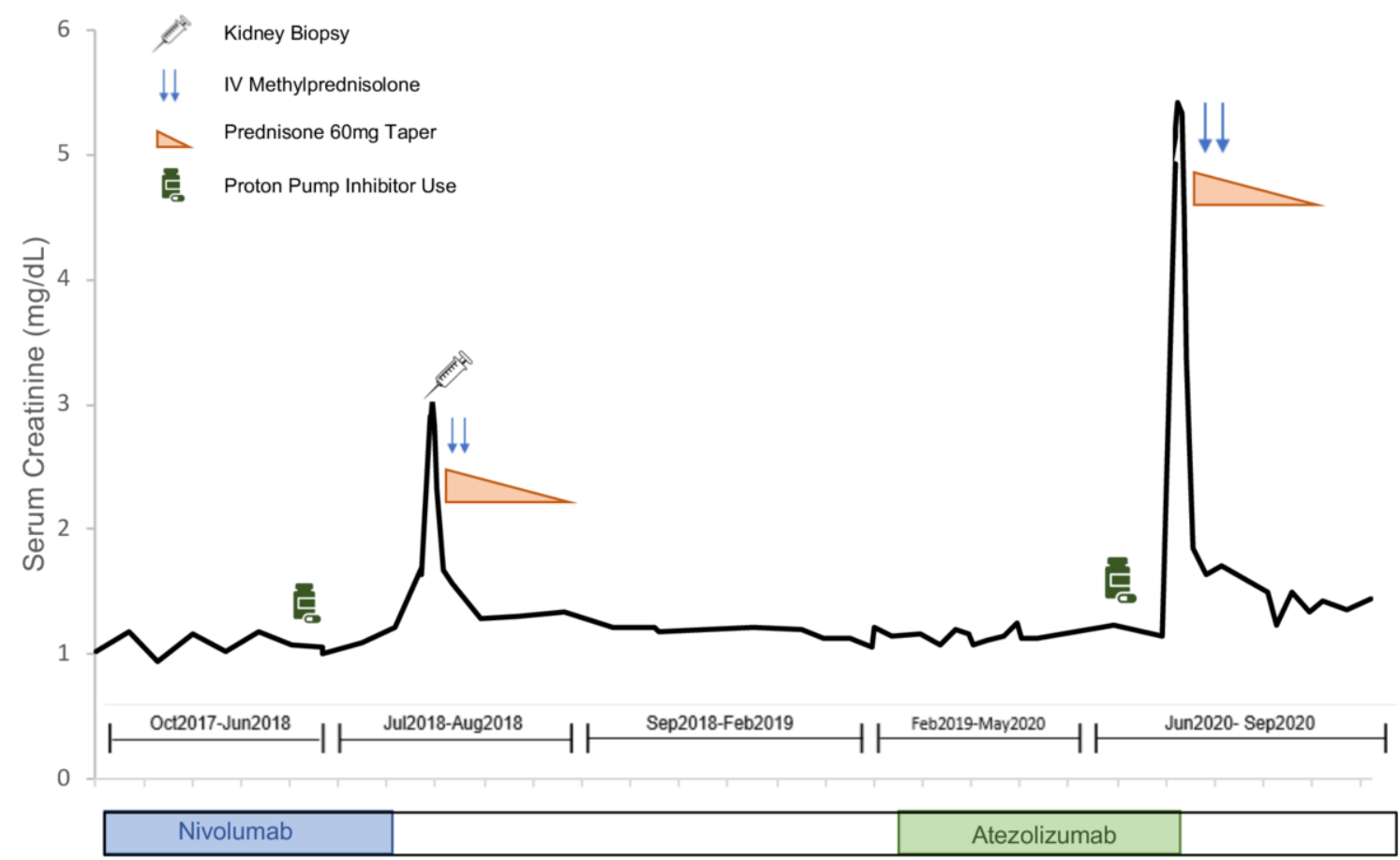

Figure 3 Clinical course of a patient who developed recurrent immune checkpoint inhibitor (ICI)-induced nephritis after reexposure to proton pump inhibitor. A patient with metastatic melanoma presented with elevated creatinine after the 8 months of nivolumab therapy (21st planned dose). He was diagnosed with $\mathrm{ICl}$-induced acute interstitial nephritis by kidney biopsy and treated by holding nivolumab (programmed death 1 inhibitor), stopping omeprazole, and with one dose of methylprednisolone $500 \mathrm{mg}$, followed by a standard of care taper of oral prednisone (beginning at $60 \mathrm{mg} /$ day and tapered to $10 \mathrm{mg}$ in $42 \mathrm{days}$ ) and he achieved renal recovery. approximately 7 months later, he experienced melanoma recurrence and was treated with atezolizumab (programmed death ligand 1 inhibitor), which he tolerated for 4 months without any adverse symptoms or kidney function abnormalities. Due to nausea, he was again prescribed omeprazole $20 \mathrm{mg} / \mathrm{day}$, and 3 weeks later was found to have a creatinine of $4.9 \mathrm{mg} / \mathrm{dL}$. Atezolizumab and omeprazole were discontinued, and he was treated with corticosteroids and again achieved renal recovery. 
that a treatment regimen with initiation of $60 \mathrm{mg}$ (or $1 \mathrm{mg} / \mathrm{kg}$ ) prednisone equivalent per day and rapidly tapering to $10 \mathrm{mg} /$ day within 3 weeks paired with discontinuation of other AIN-associated medications was equivalent at inducing renal recovery of ICI-induced AIN when compared with the NCCN guideline-based approach, which recommends an initial dose of $1-2 \mathrm{mg} / \mathrm{kg}$ prednisone equivalent per day for high-grade ICI-induced nephritis until creatinine returns to $<1.5$-fold baseline followed by a prolonged taper over $4-6$ weeks. Multiple prior studies have shown that $>85 \%-90 \%$ of patients with ICI-induced nephritis will recover with corticosteroids ${ }^{1-3}{ }^{5-8}$; however, our study used significantly shorter course of corticosteroids than all prior series. Cortazar and colleagues summarized findings from 18 centers and found that among 119 patients with ICI-induced nephritis who were treated with corticosteroids, there was no difference in the dose or duration of corticosteroids prescribed to patients with complete renal recovery compared with those with partial or no remission. However, on average, patients received longer courses of steroids. In this multicenter series, patients received greater than $20 \mathrm{mg}$ /day of prednisone equivalent for a median of 28 days (IQR 16-47) and a median of 63 total days of oral corticosteroids (IQR 32-107), suggesting that most centers were following NCCN guidelines. ${ }^{1}$ In the current series, more patients in the rapid-taper steroid group experienced renal recovery by 30 days compared with the group that received standard of care, despite receiving less corticosteroids. A possible explanation could be that the rapidtaper group had less stage $3 \mathrm{AKI}$, suggesting these cases may have been less severe or may have been caught and treated earlier. Another possible explanation is that the standard of care corticosteroid group was more likely to remain on or begin AIN-associated medications (such as PPI or trimethoprim-sulfamethoxazole) during their corticosteroid taper. Our series highlights the importance of discontinuing other AIN-associated medications, which was evident in a compelling case where recurrent AKI occurred 3 weeks after starting omeprazole in a patient who had previously been successfully tolerating ICI rechallenge. Multiple prior studies have demonstrated the association between AIN-associated medications and development of ICI-induced nephritis. ${ }^{16712}$

Outside of the cancer immunotherapy realm, the most common cause of AIN is medication-induced, and the mainstay of therapy is discontinuation of the causative drug. Initiation of corticosteroids is reserved for those with severe kidney injury or for those who fail to improve with discontinuation of offending agent. Recommendations for medication-induced AIN management are based on low quality data, and there remains considerable equipoise regarding the effectiveness of corticosteroids, with observational studies yielding conflicting results. ${ }^{13-17}$

The argument to minimize corticosteroid exposure after ICIs stems from the additional concern that highdose corticosteroids could blunt the T-cell mediated antitumor response. Patients with irAEs may have better overall survival than patients receiving ICIs who do not develop irAEs. ${ }^{18-20}$ Recent studies report that among patients with irAEs, high-dose corticosteroids are associated with decreased progression-free survival. ${ }^{21-25}$ Furthermore, pre-existing corticosteroid use has been correlated with lower cancer response rates to ICIs. ${ }^{22}$ Thus, implementing corticosteroid-minimizing strategies that allow the patient to remain on, or quickly return to ICI therapy is important. We did not evaluate and compare corticosteroid side effects between groups, and it is important to note that even short-term corticosteroid use can have adverse effects, including but not limited to hyperglycemia, gastrointestinal hemorrhage, and psychological disturbances. ${ }^{26}$ However, a rapid corticosteroid taper could improve quality of life, decreasing pill burden by avoiding need for coadministration of $P$. jirovecii prophylaxis and decrease anxiety by shortening the period of time where cancer therapy is held. ${ }^{4}$ It is important to note that more than half of patients in this series were rechallenged with ICIs after achieving renal recovery; of those rechallenged, approximately half experienced clinical benefit. The decision to rechallenge a patient after an irAE is controversial. Although NCCN guidelines recommend permanently discontinuing ICIs in high grade ICI-induced nephritis, there is increasing evidence that some previously non-responding patients may experience treatment response to a rechallenge. ${ }^{27} 28$ There is also growing evidence that ICIs can be tolerated in the majority of patients who recover from ICI-induced nephritis, with nephritis recurrence occurring in approximately 25\%. ${ }^{122930}$ This study highlights the importance of discontinuation of other AIN-associated medications when considering ICI rechallenge.

Our study has important limitations. First, this is a retrospective study and outcomes were adjudicated by chart review. To minimize selection bias, we determined the treatment group based on the initially planned corticosteroid taper, not by the ultimate duration of corticosteroids received since patients with "slow to resolve" ICI-induced nephritis are likely to receive the longest duration. Because the majority of patients were treated in our onconephrology clinic, we were able to adjudicate the planned corticosteroid course. Our change in protocol for rapid corticosteroid taper for ICI-induced AIN began in 2018; therefore, these cases were more recent and had shorter follow-up. Furthermore, history bias may have led to two important differences between the rapid-taper group and the historical controls. First, it is possible that the increased awareness of ICI-induced nephritis in these more recent cases (post-2018) led to more prompt recognition and treatment, leading to improved responses. Second, the time to rechallenge may have been longer in the historical control patients treated with standard of care corticosteroids due to greater hesitancy to rechallenge with ICIs after irAE in earlier years. Also, it is possible that as time elapses, there will be more patients rechallenged with ICIs and that relapses or recurrence of new irAEs may be more likely in these patients as they 
are followed for longer periods of time. Indeed, Cortazar and colleagues showed that the shorter the interval to ICI rechallenge, the higher the rate of relapse of ICI-induced nephritis. ${ }^{1}$ Future research is needed to identify predictors of irAE relapse. We used strict creatinine cut-offs to stage AKI and to define renal recovery. However, "refractory nephritis" among those whose creatinine did not return to within 1.5-fold baseline was not confirmed in any cases by repeat biopsy. Approximately $30 \%$ of patients had biopsy-confirmed AIN, thus, some patients could have had alternative causes of AKI such as acute tubular necrosis. However, this mirrors clinical practice, where the diagnosis of ICI-induced nephritis is often made clinically, and patients are increasingly empirically treated with corticosteroids without kidney biopsy-confirmed diagnosis. In all cases, ICI-induced nephritis was highest on the differential of the treating provider, and all but one patient was seen by a nephrologist. Although use of concomitant nephrotoxic chemotherapies was rare in this series (table 1), diagnostic ambiguity surrounding AKI after ICIs is growing as treatment regimens increasingly pair ICIs with nephrotoxic chemotherapies and targeted antineoplastic therapies. This highlights the importance of kidney biopsy in cases of diagnostic uncertainty. The risk of empiric treatment is overdiagnosis of AIN leading to ICI treatment delays and overuse of corticosteroids. This could also be minimized by adopting a rapid corticosteroid taper protocol. We excluded patients with kidney biopsies showing alternative causes of kidney pathology, so our findings do not apply to glomerular diseases that result from ICIs. ${ }^{31}$ We excluded patients with other potentially life-threatening irAEs such as myocarditis, hepatitis, pneumonitis, or neurological irAEs, as the corticosteroid strength and duration is driven by specialists and oncologists managing these toxicities these irAEs are more commonly refractory to corticosteroids, and these events may be fatal. No patients required dialysis at the time of diagnosis of ICI-induced nephritis; thus, these results cannot be generalized to cases of ICI-induced nephritis severe enough to warrant dialysis at presentation. Finally, this was sourced from a single cancer center and is a predominantly white population, which limits generalizability.

Our series suggests that patients with ICI-induced nephritis may have acceptable kidney outcomes with rapid corticosteroid taper when combined with discontinuation of other AIN-associated medications. Prospective, multicenter studies will be needed to validate these findings in larger numbers of patients. Future studies should focus on identifying baseline factors that predict corticosteroid refractoriness and relapse in patients with ICI-induced nephritis in order to allow for personalized approaches to initial management and decisions about rechallenging with ICIs. Strategies that can minimize corticosteroid exposure, lead to acceptable kidney outcomes, and allow for continuation of potentially lifesaving ICI therapy will ultimately improve outcomes for patients with metastatic cancer.
Contributors Research idea and study design: MDL, HS, KLR, MES; data acquisition: MDL, HS, IAS, MES; data analysis/interpretation: MDL, HS, IAS, RS, GMB, RMF, KLR, SHZ, MES; statistical analysis: SHZ, MES; supervision or mentorship: MES; MDL, and HS contributed equally to this work.

Funding The authors have not declared a specific grant for this research from any funding agency in the public, commercial or not-for-profit sectors.

Competing interests RS: consulting/advisory boards: Asana Biosciences, AstraZeneca, Eisai, Merck, Novartis, OncoSec, Pfizer, Replimune. Research funding: Merck. RF: consulting/advisory board: Apricity Health. GMB: advisory board: Nektar Therapeutics, Novartis; sponsored research agreements: Olink Proteomics, Palleon Pharmaceuticals; speaker: Novartis.

\section{Patient consent for publication Not required.}

Ethics approval This protocol for retrospective data collection was approved by our hospital's institutional review board, and the need for informed consent was waived.

Provenance and peer review Not commissioned; externally peer reviewed.

Data availability statement Data are available upon reasonable request.

Open access This is an open access article distributed in accordance with the Creative Commons Attribution Non Commercial (CC BY-NC 4.0) license, which permits others to distribute, remix, adapt, build upon this work non-commercially, and license their derivative works on different terms, provided the original work is properly cited, appropriate credit is given, any changes made indicated, and the use is non-commercial. See http://creativecommons.org/licenses/by-nc/4.0/.

\section{ORCID iDs}

Meghan D Lee http://orcid.org/0000-0002-6895-5968

Ryan Sullivan http://orcid.org/0000-0001-5344-6645

\section{REFERENCES}

1 Cortazar FB, Kibbelaar ZA, Glezerman IG, et al. Clinical features and outcomes of immune checkpoint inhibitor-associated AKI: a multicenter study. J Am Soc Nephrol 2020;31:435-46.

2 Brahmer JR, Lacchetti C, Schneider BJ, et al. Management of immune-related adverse events in patients treated with immune checkpoint inhibitor therapy: American Society of clinical oncology clinical practice guideline. J Clin Oncol 2018;36:1714-68.

3 Puzanov I, Diab A, Abdallah K, et al. Managing toxicities associated with immune checkpoint inhibitors: consensus recommendations from the Society for immunotherapy of cancer (SITC) toxicity management Working group. J Immunother Cancer 2017;5:95.

4 Thompson JA, Schneider BJ, Brahmer J, et al. NCCN guidelines insights: management of Immunotherapy-Related toxicities, version 1.2020. J Natl Compr Canc Netw 2020;18:230-41.

5 Cortazar FB, Marrone KA, Troxell ML, et al. Clinicopathological features of acute kidney injury associated with immune checkpoint inhibitors. Kidney Int 2016;90:638-47.

6 Seethapathy H, Zhao S, Chute DF, et al. The incidence, causes and risk factors of acute kidney injury in patients receiving immune checkpoint inhibitors. Clin J Am Soc Nephrol 2019;14:1692-700.

7 Manohar S, Ghamrawi R, Chengappa M, et al. Acute interstitial nephritis and checkpoint inhibitor therapy. Kidney360 2020;1:16-24.

8 Meraz-Muñoz A, Amir E, Ng P, et al. Acute kidney injury associated with immune checkpoint inhibitor therapy: incidence, risk factors and outcomes. J Immunother Cancer 2020;8:e000467.

9 Liu D, Ahmet A, Ward L, et al. A practical guide to the monitoring and management of the complications of systemic corticosteroid therapy. Allergy Asthma Clin Immunol 2013;9:30.

10 Eisenhauer EA, Therasse P, Bogaerts J, et al. New response evaluation criteria in solid tumours: revised RECIST guideline (version 1.1). Eur J Cancer 2009;45:228-47.

$11 \mathrm{KDIGO}$ clinical practice guideline for acute kidney injury. Kidney International 2012;2:138.

12 Seethapathy H, Zhao S, Strohbehn IA, et al. Incidence and clinical features of immune-related acute kidney injury in patients receiving programmed cell death ligand-1 inhibitors. Kidney Int Rep 2020;5:1700-5

13 González E, Gutiérrez E, Galeano C, et al. Early steroid treatment improves the recovery of renal function in patients with drug-induced acute interstitial nephritis. Kidney Int 2008;73:940-6.

14 Handa SP. Drug-Induced acute interstitial nephritis: report of 10 cases. CMAJ 1986;135:1278-81.

15 Buysen JG, Houthoff HJ, Krediet RT, et al. Acute interstitial nephritis: a clinical and morphological study in 27 patients. Nephrol Dial Transplant 1990;5:94-9. 
16 Schwarz A, Krause PH, Kunzendorf U, et al. The outcome of acute interstitial nephritis: risk factors for the transition from acute to chronic interstitial nephritis. Clin Nephrol 2000;54:179-90.

17 Muriithi AK, Leung N, Valeri AM, et al. Biopsy-proven acute interstitial nephritis, 1993-2011: a case series. Am J Kidney Dis 2014;64:558-66.

18 Shankar B, Zhang J, Naqash AR, et al. Multisystem immune-related adverse events associated with immune checkpoint inhibitors for treatment of non-small cell lung cancer. JAMA Oncol 2020;6:1952.

19 Matsuoka H, Hayashi T, Takigami K, et al. Correlation between immune-related adverse events and prognosis in patients with various cancers treated with anti PD- 1 antibody. BMC Cancer 2020;20:656.

20 Das S, Johnson DB. Immune-Related adverse events and anti-tumor efficacy of immune checkpoint inhibitors. J Immunother Cancer 2019;7:306

21 X SL B, Guo J, et al. Correlation between system-specific irAEs, associated glucocorticoid exposure, and antiPD-1 monotherapy efficacy in advanced melanoma. Society for Melanoma Research 2019 Abstract 2019.

22 Arbour KC, Mezquita L, Long N, et al. Impact of baseline steroids on efficacy of programmed cell death-1 and programmed Death-Ligand 1 blockade in patients with non-small-cell lung cancer. J Clin Oncol 2018;36:2872-8.

23 Hughes MS, Molina GE, Chen ST, et al. Budesonide treatment for microscopic colitis from immune checkpoint inhibitors. J Immunother Cancer 2019;7:292.
24 Faje AT, Lawrence D, Flaherty K, et al. High-Dose glucocorticoids for the treatment of ipilimumab-induced hypophysitis is associated with reduced survival in patients with melanoma. Cancer 2018;124:3706-14.

25 Petrelli F, Signorelli D, Ghidini M, et al. Association of steroids use with survival in patients treated with immune checkpoint inhibitors: a systematic review and meta-analysis. Cancers 2020;12. doi:10.3390/ cancers12030546

26 Yao T-C, Huang Y-W, Chang S-M, et al. Association Between Oral Corticosteroid Bursts and Severe Adverse Events : A Nationwide Population-Based Cohort Study. Ann Intern Med 2020;173:325-30.

27 Santini FC, Rizvi H, Plodkowski AJ, et al. Safety and efficacy of Retreating with immunotherapy after immune-related adverse events in patients with NSCLC. Cancer Immunol Res 2018;6:1093-9.

28 Abou Alaiwi S, Xie W, Nassar AH, et al. Safety and efficacy of restarting immune checkpoint inhibitors after clinically significant immune-related adverse events in metastatic renal cell carcinoma. $J$ Immunother Cancer 2020;8:e000144.

29 Johnson DB, Jakubovic BD, Sibaud V, et al. Balancing cancer immunotherapy efficacy and toxicity. J Allergy Clin Immunol Pract 2020;8:2898-906.

30 Thompson JA, Schneider BJ, Brahmer J, et al. Management of Immunotherapy-Related toxicities, version 1.2019. J Natl Compr Canc Netw 2019;17:255-89.

31 Mamlouk O, Selamet U, Machado S, et al. Nephrotoxicity of immune checkpoint inhibitors beyond tubulointerstitial nephritis: single-center experience. J Immunother Cancer 2019;7:2. 